\title{
Os desafios dos cuidados paliativos na atenção primária à saúde
}

\author{
The challenges of palliative care in primary health care
}

Los desafíos de los cuidados paliativos en la atención primaria de salud

Laura Comeli Ordonho ${ }^{1 *}$, Isadora Correia Dias², Julia de Oliveira Bernardino ${ }^{3}$, Jullyana Lopes Almeida ${ }^{4}$, Lucio Mendes Junior ${ }^{5}$, Mariana Martins de Mello Paula ${ }^{6}$, Marina Perigolo Barros Quintão ${ }^{7}$, Nayara Lima de Miranda ${ }^{8}$, Talita de Oliveira Côrtes Mendes ${ }^{9}$, Mônica Isaura Corrêa ${ }^{7}$.

\section{RESUMO}

Objetivo: Revisar e descrever o papel da Atenção Primária em Saúde (APS) no manejo ao paciente em Cuidado Paliativo (CP), a partir da análise da literatura atual sobre o tema. Revisão bibliográfica: O CP está associado à melhora da qualidade de vida de pacientes que enfrentam doenças graves e de seus familiares. Nesse contexto, a APS é vista como o melhor local para a prática do $\mathrm{CP}$, uma vez que tem como objetivo o cuidado integral do paciente e sua família, a partir de uma relação mais próxima com a equipe de saúde. Entretanto, o CP ainda é um desafio para o Sistema Único de Saúde devido, principalmente, à alta demanda decorrente do envelhecimento populacional e aumento dos agravos crônicos, a não disponibilidade de recursos tecnológicos e humanos capacitados e a escassez de programas governamentais específicos. Considerações finais: É de suma importância que haja a implementação de diretrizes e protocolos de saúde voltadas para desenvolver os $\mathrm{CP}$, maior capacitação profissional e estímulo para maior consciência social e envolvimento da comunidade como um todo com as questões de saúde da população.

Palavras-chave: Cuidados paliativos, Atenção primária à saúde, Assistência domiciliar.

\begin{abstract}
Objective: Review and describe the role of Primary Health Care (PHC) in patient management in Palliative Care (PC), based on an analysis of the current literature on the subject. Bibliographic review: PC is associated with an improvement in the quality of life of patients who face serious illnesses and their families. In this context, PHC is seen as the best place to practice PC, as it aims to provide comprehensive care for patients and their families, based on a closer relationship with the health team. However, PC is still a challenge for the Unified Health System, mainly due to the high demand resulting from population aging and the increase in chronic diseases, the non-availability of trained technological and human resources and the scarcity of specific government programs. Final considerations: It is extremely important to implement health guidelines and protocols aimed at developing PCs, greater professional training and encouragement for greater social awareness and involvement of the community as a whole with the population's health issues.
\end{abstract}

Key words: Palliative care, Primary health care, Home nursing.

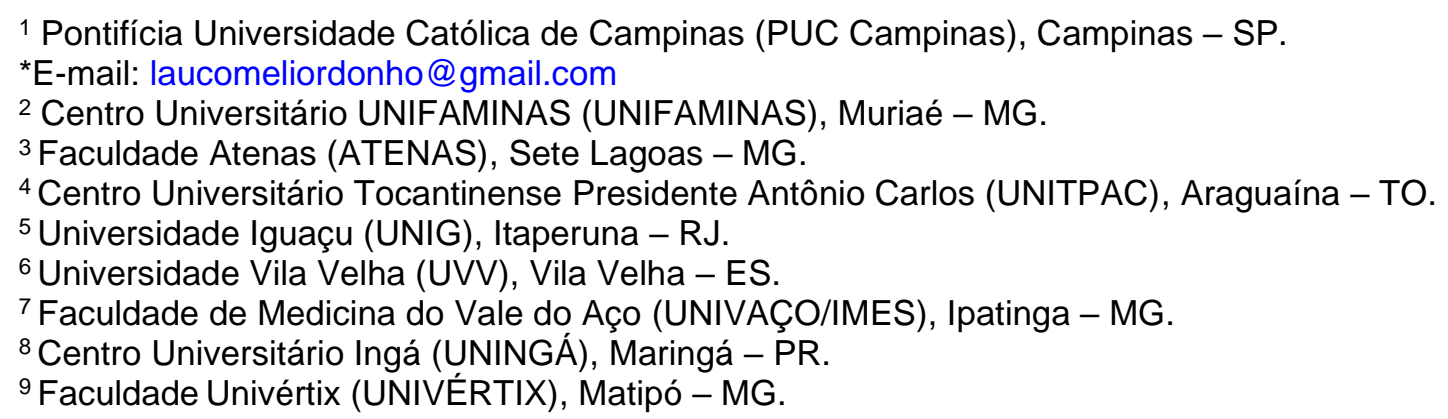




\section{RESUMEN}

Objetivo: Revisar y describir el papel de la Atención Primaria de Salud (APS) en el manejo del paciente en Cuidados Paliativos (CP), a partir de un análisis de la literatura actual sobre el tema. Revisión bibliográfica: Los CP se asocian con una mejora en la calidad de vida de los pacientes que enfrentan enfermedades graves y sus familias. En este contexto, la APS es vista como el mejor lugar para la práctica de CP, ya que tiene como objetivo brindar una atención integral al paciente y su familia, basada en una relación más cercana con el equipo de salud. Sin embargo, la CP sigue siendo un desafío para el Sistema Único de Salud, principalmente por la alta demanda derivada del envejecimiento de la población y el aumento de enfermedades crónicas, la no disponibilidad de recursos humanos y tecnológicos y la escasez de programas gubernamentales específicos. Consideraciones finales: Es de suma importancia la implementación de guías y protocolos de salud dirigidos al desarrollo de CP, mayor formación profesional y estímulo para una mayor conciencia social e involucramiento de la comunidad en su conjunto con los problemas de salud de la población.

Palabras clave: Atención primaria de salud, Atención domiciliaria de salud, Cuidados en el hogar.

\section{INTRODUÇÃO}

O Cuidado Paliativo (CP) baseia-se na assistência multidisciplinar prestada a pacientes e familiares com objetivo principal de oferecer qualidade de vida, por meio da prevenção e alívio do sofrimento, aos portadores de uma doença que ameace a continuidade da vida (MINISTÉRIO DA SAÚDE, 2020). Dessa forma, os CP devem ser centrados na pessoa, valorizando suas necessidades, de forma que o mesmo receba informações verdadeiras sobre seu estado de saúde e que ele participe ativamente nas tomadas de decisão sobre o tratamento recebido (ORGANIZAÇÃO MUNDIAL DA SAÚDE (OMS), 2014).

Atualmente a demanda por CP configura-se um problema de saúde pública em virtude do aumento da expectativa de vida e, por consequência, o aumento de doenças crônicas-degenerativas não transmissíveis no Brasil e no mundo (EVELINE TJ, et al., 2020). Dessa forma, embora essa assistência seja oferecida mais comumente a pacientes com neoplasias, várias outras Doenças Crônicas (DC) são englobadas por esse cuidado como a tuberculose resistente, insuficiência cardíaca, doença pulmonar obstrutiva crônica, doença de Alzheimer, Síndrome da Imunodeficiência Adquirida (AIDS) e outras patologias (SAMI AA, et al., 2020; CISSA A, 2017).

Segundo a Academia Nacional de Cuidados Paliativos (ANCP), os CP foram difundidos pelo mundo principalmente na segunda metade do século XX, mas foi na década de 1980 que essa assistência começou a ser implantada no Brasil. Até então, seu propósito era baseado em oferecer um cuidado total e ativo para pacientes com doenças incuráveis. Entretanto, a partir dos anos 2000, a Organização Mundial da Saúde (OMS) modifica esse conceito, tirando o foco da dualidade de doenças curáveis/incuráveis para uma abordagem focada no sofrimento e independente do prognóstico (ANCP, 2018; OMS, 2014).

No Brasil, somente em 2011 os Cuidados Paliativos foram reconhecidos como uma subespecialidade médica pelo Conselho Federal de Medicina e tornou-se uma área de atuação possível para seis especialidades, sendo elas: geriatria, pediatria, oncologia, clínica médica, anestesia e medicina de família (FIGUEIREDO MC e STANO RT, 2013). Em 2019, o país contava com 190 serviços de CP, número que vem crescendo anualmente. Porém, constata-se uma desigualdade de disponibilidade, visto que mais de $50 \%$ dos serviços estão concentrados na região sudeste, de forma que essa oferta ainda é insuficiente para colocar o país no grupo de nações com melhor nível de cobertura nessa área (SANTOS AJ, et al., 2020; ANCP, 2018).

As ações em CP podem ser prestadas por meio de três modelos de assistência à saúde: hospitalar, ambulatorial e domiciliar. Cada um tem suas vantagens e desvantagens, mas quando se pensa apenas na atenção domiciliar, deve-se considerar um cuidado que permite o acolhimento do paciente em seu próprio lar, junto à sua família, sem ter que seguir uma rotina hospitalar (ANCP, 2018). Neste contexto, a Atenção Primária em Saúde (APS) é vista como o melhor local para a prática do CP, uma vez que tem como objetivo o cuidado 
integral do paciente e sua família, a partir de uma relação mais próxima com a equipe de saúde (JÚLIA RR e KÁTIA P, 2019).

Entretanto, é um grande desafio para o Sistema Único de Saúde brasileiro (SUS), bem como para a APS em si, se organizar a fim de atender às atuais demandas de saúde de uma população idosa e com tantos agravos crônicos. Ao analisar a história do SUS e dos serviços de saúde no país, conclui-se que sua organização sempre foi voltada ao atendimento às condições agudas ou episódios de agudização de enfermidades crônicas (MENDES EV, 2012).

Diante do exposto, o objetivo deste trabalho foi revisar e descrever o papel da APS no manejo ao paciente em cuidado paliativo, a partir da análise da literatura atual sobre o tema.

\section{REVISÃO BIBLIOGRÁFICA}

O termo Cuidado Paliativo foi definido pela primeira vez na década de 90, pela OMS e baseava-se em um cuidado ativo e total, englobando o alívio da dor e de questões psíquicas, espirituais e sociais, que se direcionava aos pacientes com doenças incuráveis. A limitação do público alvo desse manejo, a apenas pacientes com doenças terminais, dificultou sua aplicação adequada, além de fortalecer a visão dicotômica que dividia os pacientes em "curáveis" e "não curáveis" (GOMES AZ e OTHERO MB, 2016).

No ano de 2002, OMS redefiniu o termo "Cuidados Paliativos" como sendo uma abordagem com o objetivo de melhorar a qualidade de vida tanto dos pacientes que enfrentam doenças que representam uma ameaça à sobrevivência, quanto de seus familiares, amigos e cuidadores, ampliando, portanto, a definição anterior. Essa abordagem prevê um enfoque maior no bem-estar do paciente, e não somente na cura e no tratamento da doença em si. Dessa forma, a implementação dos CP deve ser realizada o mais precoce possível, juntamente com os tratamentos modificadores da patologia (OMS, 2014).

A definição mais recente de "Cuidados Paliativos" foi publicada no ano de 2018 pela International Association for Hospice \& Palliative Care (IAHPC), e esse conceito se assemelha àquele estabelecido no ano de 2002, mantendo a prioridade no alívio do sofrimento daqueles pacientes em situação de doença grave. Desta forma, qualquer pessoa detentora de uma doença crônica ou que ameace a vida, seja ele criança, adulto ou idoso, é passível de receber cuidados paliativos (JUSTINO ET, et al., 2020).

O manejo do indivíduo em CP envolve o respeito à autonomia e poder de escolha do paciente, além da avaliação precoce, controle e alívio da dor e de outros problemas, sejam eles físicos, psicossociais e espirituais. Essa abordagem engloba, ainda, a oferta de um sistema de apoio que tem por finalidade auxiliar as pessoas próximas do paciente a lidar com o processo de adoecimento e com o luto pós óbito. Assim, a prática dos $\mathrm{CP}$ se configura, necessariamente, como um trabalho de equipe de caráter interprofissional, que conta com médicos, enfermeiros e técnicos de enfermagem, assistentes sociais, psicólogos, farmacêuticos, fisioterapeutas, terapeutas ocupacionais, nutricionistas e assistentes espirituais de caráter ecumênico ou da religião escolhida pelo paciente (SAGA Y, et al., 2018; GOMES AZ e OTHERO MB, 2016).

Atualmente, existe uma demanda crescente por CP na saúde pública, o que faz o tema ser de extrema relevância diante do progressivo envelhecimento populacional mundial, que tem por consequência 0 crescimento do número de idosos e aumento da incidência das doenças crônico-degenerativas não transmissíveis (SOUZA HL, et al., 2015). De acordo com as estimativas globais, as principais patologias que necessitam de cuidados paliativos são as doenças cardiovasculares (38\%), seguida pelas neoplasias (34\%), Doença Pulmonar Obstrutiva Crônica (DPOC) (10\%) e a AIDS (10\%) (WORLDWIDE PALLIATIVE CARE ALLIANCE, 2014).

No entanto, não há acesso suficiente aos CP em todo o mundo, especialmente em países de média e baixa renda. De acordo com o levantamento feito pela ANCP, em 2018, existiam 177 serviços de CP no Brasil, entretanto, mais de 50\% desses serviços iniciaram tardiamente suas atividades, por volta do ano de 2010 , reforçando o quanto essa abordagem é recente no país. Além disso, essa pesquisa também mostrou que esses cuidados estão centrados em hospitais, porém menos de $10 \%$ dos hospitais do país apresentam uma equipe de CP (MARCUCCI FI, et al., 2016; ANCP, 2018). 
A Associação Européia de Cuidados Paliativos sugere a existência de dois serviços de CP para cada 100.000 habitantes. Entretanto, essa proposta não é atendida no cenário brasileiro, uma vez que, considerando uma população de aproximadamente 210 milhões de habitantes, verifica-se que há cerca de um serviço desse tipo para cada 1,1 milhão de pessoas, e que o acesso a essa abordagem no sistema público de saúde está concentrado em centros especializados em câncer e iniciativas locais (ANCP, 2018).

Tendo em pauta os princípios do SUS, que determinam a saúde como um direito fundamental a todos os cidadãos, foram promulgadas no ano de 2002 a Portaria no 19, que instituiu um Programa Nacional de Assistência à Dor e Cuidados Paliativos dentro do SUS, e a Lei № 10.424, regulamentando o atendimento e internação domiciliar no SUS. Embora os CP tenham sido incluídos como parte dessas iniciativas, ainda não estavam amplamente disponíveis (RIBEIRO JR e POLES K, 2019; MARCUCCI FI, et al., 2016). Somente em 2018, o Ministério da Saúde publicou a Resolução no 41, que normatiza a oferta de CP como parte dos cuidados continuados integrados no âmbito do SUS e definindo que estes devem estar disponíveis em todo ponto da rede, desde a atenção básica, domiciliar, ambulatorial, até os serviços hospitalares e de urgência e emergência (MINISTÉRIO DA SAÚDE, 2018).

Nesse contexto, sabe-se que a APS é definida como sendo o primeiro nível de contato dos indivíduos, da família e da comunidade com a saúde, baseada em um conjunto de ações individuais e coletivas que envolvem a promoção, prevenção, proteção e cuidado integrado dirigido à população em território definido. Assim, este primeiro nível de atenção configura-se como um importante mecanismo para a implantação de $\mathrm{CP}$, tendo importância desde o início do tratamento de uma doença, até o planejamento e conduta de pacientes paliativos, garantindo melhor qualidade de vida e suporte às famílias, além da oferta de uma variedade de serviços de saúde, muitos dos quais são necessários enquanto o paciente permanece morando na comunidade (GUSSO G e LOPES JC, 2019; ETKIND SN, et al., 2018; MARCUCCI FI, et al., 2016; JÚLIA RR e KÁTIA P, 2019).

Dessa forma, estudos têm demonstrado que a implementação dos CP na APS está associada com melhora do controle dos sintomas, redução no número de internações hospitalares, maior satisfação do paciente e familiares e redução de custos quando comparados aos cuidados empregados na atenção especializada, além de reduzir a sobrecarga dos outros serviços de saúde. Esses benefícios ocorrem uma vez que, quando já existe um acompanhamento do paciente desde o início do tratamento de determinada patologia na APS, podem ser elaborados planos de ação, junto ao doente e a família, que poderão ser aplicados tornando assim mais natural o tratamento paliativo (MELO CM, et al., 2021).

A população idosa é o grupo com maior necessidade de CP, especialmente em virtude de tratamentos crônicos e de longa duração como em doenças como a demência, câncer, doenças cardiovasculares, doenças respiratórias e doenças renais (AZEVEDO C, et al., 2016). Inicialmente, os CP eram voltados para os portadores de câncer, preconizando os cuidados de final de vida. Com a revisão e ampliação do conceito ao longo dos anos, recomendou-se a assistência às outras doenças, reforçando a necessidade de incluir os $\mathrm{CP}$ como parte da assistência completa à saúde, no tratamento a todas as doenças crônicas e em programas de atenção aos idosos (GOMES AZ e OTHERO MB, 2016).

Pesquisas estimam que, anualmente, cerca de 20 milhões de pessoas ao redor do mundo necessitam de CP, sendo a maior parte destes idosos (AZEVEDO C, et al., 2016). Um estudo realizado no estado do Paraná estimou que o número de pacientes que necessitam de CP registrados na APS no Brasil é entre 456 e 537 para cada 100.000 habitantes, sendo que a demência e as doenças cardiovasculares foram as condições mais frequentes naqueles pacientes identificados como necessitando de CP. Assim, considerando as dimensões continentais de um país como o Brasil, conclui-se que hoje existe um número muito grande de pessoas que carecem destes cuidados (MARCUCCI FI, et al., 2016).

Marcucci FI, et al. (2016) observaram, ainda, que o acesso a abordagens multiprofissionais oferecidas por ambientes de cuidados primários foi muito limitado entre os pacientes avaliados e que o suporte profissional da atenção básica a pacientes em CP é prestado principalmente por equipe de enfermagem, médicos generalistas e agentes comunitários de saúde. Entretanto, reforça-se a importância da disponibilidade de uma equipe composta por outros profissionais como assistentes sociais, nutricionistas, psicólogos, entre outros, 
para um serviço completo, que seja capaz de abarcar desde a terapia física à psicoterapia (MELO CM et al., 2021). No estudo em questão, apenas $10 \%$ dos pacientes participantes receberam fisioterapia, e nenhum recebeu consulta de psicologia (MARCUCCI FI, et al., 2016).

Entre os pressupostos para uma adequada prática de CP está o início precoce do acompanhamento em $\mathrm{CP}$, incluindo investigação necessária para compreender qual a melhor tratamento e conduta frente aos sintomas apresentados; compreensão sobre a importância da vida, mas compreendendo a morte como um processo natural; promoção da avaliação, reavaliação e alívio de situações que ocasionam a dor ou desconforto e; acesso à equipe multidisciplinar de forma a atender o indivíduo de forma completa além dos aspectos físicos e espirituais. Diante dessas ações, o paciente receberá o melhor suporte com o objetivo de promover melhora da qualidade de vida e auxiliando-o a viver tão ativamente quanto possível até a sua morte (MINISTÉRIO DA SAÚDE, 2020).

Ainda que os CP na APS não deva ser promovida como um tipo "internação domiciliar", as ações da APS por si já preveem visitas de dos profissionais ao domicílio, de modo que, mesmo não tendo sido originalmente desenvolvidos para ações de CP, podem ser estruturados a fim de incorporar tal modelo, se tornando uma importante ferramenta nessa modalidade de cuidado. Outra iniciativa do Ministério da Saúde que é suporte nos cuidados domiciliares é o Serviço de Atenção Domiciliar Melhor em Casa que é um programa de atendimento domiciliar que tem como público-alvo pessoas que necessitam de cuidados diários em domicílio, para recuperação de problema agudo de saúde (SOUZA HL, et al., 2015; MINISTÉRIO DA SAÚDE, 2017).

Dessa forma, é fundamental que esta rede de assistência seja disponível e flexível, fazendo parte desta organização a oferta e acesso rápido a medicamentos, facilidade de interconsultas e hospital de retaguarda, em caso de necessidade de internação ou realização de procedimentos de diagnóstico ou de tratamento (SOUZA HL, et al., 2015; MARCUCCI FI, et al., 2016).

Países com alto padrão de suporte em CP são caracterizados pela ampla oferta de provedores de serviços e ampla conscientização sobre CP por parte dos profissionais de saúde e da sociedade em geral; acesso irrestrito a opioides e medicamentos analgésicos; políticas públicas específicas nesta área, além de estrutura educacional e acadêmica associada ao desenvolvimento de profissionais capacitados em CP (MARCUCCI FI, et al., 2016).

Desta maneira, ao considerar o cenário brasileiro, deficiente de serviços e equipes especializadas, onde grande parte dos pacientes ainda morrem antes de ter acesso a um especialista ou generalista em cuidados paliativos, reforça-se o papel valioso da APS na condução desses cuidados (SANTOS JUNIOR AG, et al., 2016).

Ainda assim, não obstante o crescimento da demanda de CP, os serviços de APS têm tido dificuldades na oferta integral e de qualidade destas ações e ainda há muitos desafios a serem vencidos. Os principais entraves estão relacionados à complexidade dessas práticas, a não disponibilidade de recursos tecnológicos, descontinuação dos cuidados e, principalmente, na inabilidade dos profissionais para lidar com as famílias, mesmo diante da reconhecida capacidade destes para estabelecer vínculo com os pacientes que recebem tais cuidados, inabilidade essa decorrente, em grande parte, do despreparo dos profissionais desde sua formação (RIBEIRO JR e POLES K, 2019; SOUZA HL, et al., 2015; GOMES AZ e OTHERO MB, 2016; JÚLIA RR e KÁTIA P, 2019; QUEIROGA VM, et al., 2020).

Logo, ainda há muito para ser elaborado em termos de pesquisa, ensino, organização de serviços e formação de recursos humanos para que a oferta dos CP na APS não venha somente onerar ainda mais o serviço público, mas seja realizada em regime de excelência de forma a receber e tratar esses pacientes paliativos juntamente com suas famílias (TREVISAN DM, et al., 2020).

Nesse contexto, é fundamental ampliar a discussão e a formação sobre os CP, aprimorando o currículo dos cursos técnicos, de graduação e de pós-graduação, bem como a capacitação e atualização dos profissionais já em atuação, de maneira que estes estejam mais preparados e sejam multiplicadores dos CP, fortalecendo as políticas públicas e programas de humanização do cuidado em final de vida (ALVES RF, et al., 2019; VASCONCELLOS AS, et al., 2017). 


\section{CONSIDERAÇÕES FINAIS}

A partir da análise da literatura, verificou-se que há uma crescente demanda por ações de CP em todos os níveis de atenção à saúde, reforçando a importância e a urgência da sistematização de diretrizes e ações para implantação desses cuidados na APS, principalmente quando esse cuidado se dá em nível domiciliar. Entretanto, melhorias ainda são necessárias a fim de proporcionar melhor acesso e condições de cuidado ao paciente com condições limitantes de vida e sua família, como a implementação de políticas de saúde públicas voltadas para desenvolver os $\mathrm{CP}$, maior capacitação profissional, além do estímulo para maior consciência social e envolvimento da comunidade com as questões do CP.

\section{REFERÊNCIAS}

1. ACADEMIA NACIONAL DE CUIDADOS PALIATIVOS (ANCP). Panorama dos Cuidados Paliativos no Brasil. 2018. Disponível em: https://paliativo.org.br/wp-content/uploads/2018/10/Panorama-dos-Cuidados-Paliativos-no-Brasil2018.pdf. Acesso em: 29 de julho de 2021.

2. ALVES RF, et al. Cuidados Paliativos: Alternativa para o Cuidado Essencial no Fim da Vida. Psicologia: Ciência e Profissão, 2019; 39: e185734.

3. AZEVEDO C, et al. Prospects for palliative care in primary healthcare: a descriptive study. Online Brazilian Journal of Nursing, 2016; 15(4):683-693.

4. CISSA A. Interface between social support, quality of life and depression in users eligible for palliative care. Revista da Escola de Enfermagem da USP, 2017; 51: e03245.

5. ETKIND SN, et al. How many people will need palliative care in 2040 ? Past trends, future projections and implications for services. BMC Medicine, 2017;15(1): 1-09.

6. EVELINE TJ, et al. Palliative care in primary health care: scoping review. Revista Latino-Americana de Enfermagem, 2020; 28: e3324.

7. FIGUEIREDO MC, STANO RT. O Estudo da Morte e dos Cuidados Paliativos: uma Experiência Didática no Currículo de Medicina. Revista Brasileira de Educação Médica, 2013; 37(2): 298-307.

8. GOMES AZ, OTHERO MB. Cuidados paliativos. Estudos Avançados, 2016; 30(88): 155-166.

9. GUSSO G, LOPES JC. Tratado de medicina de família e comunidade. 2ª Edição. Porto Alegre: ArtMed; 2019.

10. JÚLIA RR, KÁTIA P. Cuidados paliativos: prática dos médicos da Estratégia Saúde da Família. Revista Brasileira de Educação Médica, 2019; 43(3): 62-72.

11. JUSTINO ET, et al. Os cuidados paliativos na atenção primária à saúde: scoping review. Revista Latino-Americana de Enfermagem, 2020; 28: e3324.

12. $\mathrm{MARCUCCI} \mathrm{FI}$, et al. Identification and characteristics of patients with palliative care needs in Brazilian primary care. BMC Palliative Care, 2016; 15(51): 1-10.

13. MELO CM, et al. Concepções, desafios e competências dos enfermeiros em cuidados paliativos na atenção primária à saúde. Revista Nursing, 2021; 24(277): 5833-5839.

14. MENDES EV. O cuidado das condições crônicas na atenção primária à saúde: o imperativo da consolidação da Estratégia da Saúde da Família. Organização Pan-Americana da Saúde. 2012. Disponível em: https://bvsms.saude.gov.br/bvs/publicacoes/cuidado_condicoes_atencao_primaria_saude.pdf. Acesso em: 22 de agosto de 2021.

15. MINISTÉRIO DA SAÚDE. Serviço de Atenção Domiciliar - Melhor em Casa. Brasil, 2017. Disponível em: https://www.gov.br/saude/pt-br/acesso-a-informacao/acoes-e-programas/melhor-em-casa. Acesso em: 31 de agosto de 2021.

16. MINISTÉRIO DA SAÚDE. Hospital Sírio-Libanês. Manual de Cuidados Paliativos. Brasil, 2020. Disponível em: https://www.conass.org.br/wp-content/uploads/2020/12/Manual-CuidadosPaliativos-versa\%CC\%83o-final-1.pdf. Acesso em 22 de agosto de 2021.

17. MINISTÉRIO DA SAÚDE. Resolução no 41, de 31 de outubro de 2018 - Dispõe sobre as diretrizes para a organização dos cuidados paliativos, à luz dos cuidados continuados integrados, no âmbito Sistema Único de Saúde (SUS). Brasil, 2018. Disponível em:https://www.in.gov.br/materia/-/asset_publisher/Kujrw0TZC2Mb/content/id/51520746/do1-201811-23-resolucao-n-41-de-31-de-outubro-de-2018-51520710. Acesso em: 15 de agosto de 2021.

18. ORGANIZAÇÃO MUNDIAL DA SAÚDE (OMS). Worldwide Palliative Care Aliance. Global Atlas of Palliative Care at the end of Life. Genebra, 2014. Disponível em https://www.who.int/nmh/Global_Atlas_of_Palliative_Care.pdf. Acesso em: 29 de julho de 2021.

19. QUEIROGA VM, et al. Cuidados Paliativos de Idosos no Contexto da Atenção Primária à Saúde: Uma revisão da literatura. Brazilian Journal of Developmment, 2020; 6(6):38821-38832. 
20. RIBEIRO JR, POLES K. Cuidados Paliativos: Prática dos Médicos da Estratégia Saúde da Família. Revista Brasileira de Educação Médica, 2019; 43(3):62-72.

21. SAGA Y, et al. Transitions in palliative care: conceptual diversification and the integration of palliative care into standard oncology care. Chinese Clinical Oncology, 2018; 7(3): 32.

22. SANTOS AJ, et al. Atlas dos Cuidados Paliativos no Brasil 2019. Academia Nacional de Cuidados Paliativos. 2020. Disponível em: https://api-wordpress.paliativo.org.br/wpcontent/uploads/2020/05/ATLAS_2019_final_compressed.pdf. Acesso em: 29 de julho de 2021.

23. SANTOS JUNIOR AG, et al. Desafios para os cuidados paliativos na atenção primária à saúde: Revisão integrativa de literatura. Revista de enfermagem UFPE online, 2016; 10(7): 2708-2719.

24. SAMI AA, et al. Integration of palliative care into primary health care: model of care experience. Cureus, 2020;12(6): e8866.

25. SOUZA HL, et al. Cuidados paliativos na atenção primária à saúde: considerações éticas. Revista bioética, 2015; 23(2): 349-359.

26. TREVISAN DM, et al. Perspectivas dos profissionais da saúde sobre o cuidado a pacientes em processo de finitude. Psicologia: Ciência e Profissão 2020; 40: 1-15.

27. VASCONCELOS SA, et al. Experiências vividas por enfermeiros sobre os cuidados paliativos no ambiente domiciliar. Journal Health NPEPS, 2020; 5(2): 274-290. 